\title{
Natural emergence of neutrino masses and dark matter from $R$-symmetry
}

\author{
Sabyasachi Chakraborty ${ }^{a}$ and Joydeep Chakrabortty ${ }^{b}$ \\ ${ }^{a}$ Department of Theoretical Physics, Tata Institute of Fundamental Research, \\ 1, Homi Bhabha Road, Mumbai 400005, India \\ ${ }^{b}$ Department of Physics, Indian Institute of Technology, \\ Kanpur-208016, India \\ E-mail: sabya@theory.tifr.res.in, joydeep@iitk.ac.in
}

ABStRaCt: We propose a supersymmetric extension of the Standard Model (SM) with a continuous global $\mathrm{U}(1)_{R}$ symmetry. The $R$-charges of the SM fields are identified with that of their lepton numbers. As a result, both bilinear and trilinear ' $R$-parity violating' (RPV) terms could be present at the superpotential. However, $R$-symmetry is not an exact symmetry as it is broken by supergravity effects. Hence, sneutrinos acquire a small vacuum expectation value in this framework. However, a suitable choice of basis ensures that the bilinear RPV terms can be completely rotated away from the superpotential and the scalar potential. On the other hand, the trilinear terms play a very crucial role in generating neutrino masses and mixing at the tree level. This is noticeably different from the typical $R$-parity violating Minimal Supersymmetric Standard Model. Also, gravitino mass turns out to be the order parameter of $R$-breaking and is directly related to the neutrino mass. We show that such a gravitino, within the mass range $200 \mathrm{keV} \lesssim m_{3 / 2} \lesssim 0.1 \mathrm{GeV}$ can be an excellent dark matter candidate. Finally, we looked into the collider implications of our framework.

KEYWORDS: Supersymmetry Phenomenology

ARXIV EPRINT: 1701.04566 


\section{Contents}

1 Introduction 1

2 The model 3

2.1 Soft (super-soft) SUSY breaking interactions 4

$2.2 \quad R$-symmetry breaking 5

2.3 Sneutrino VEV 6

$\begin{array}{ll}2.4 \text { Choice of basis } & 7\end{array}$

3 The fermion sector $\quad 8$

3.1 The neutral fermion sector $\quad 9$

$\begin{array}{ll}3.2 & \text { Neutrino mass and mixing } \\ 3.30\end{array}$

$\begin{array}{lll}3.3 & \text { Charged fermion sector } & 12\end{array}$

4 Gravitino as dark matter 13

4.1 Production of gravitinos 13

$\begin{array}{ll}4.2 \text { Gravitino decay width and life-time } & 14\end{array}$

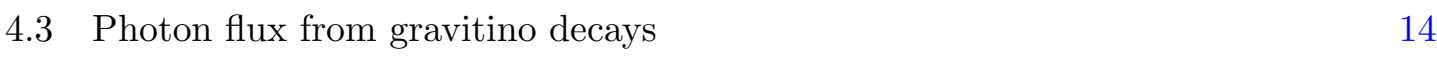

5 Collider phenomenology 16

$\begin{array}{lll}6 & \text { Conclusion } & 17\end{array}$

\section{Introduction}

Neutrino oscillation experiments have firmly established the existence of tiny non-zero masses of active neutrinos and non-trivial mixing [1] in the lepton sector. Since neutrinos are massless within the paradigm of Standard Model (SM), therefore, neutrino physics is a natural testing ground for physics beyond the SM (BSM). The most popular way to generate neutrino masses is through the see-saw mechanism. This also predicts Majorana nature of the neutrinos which signifies lepton number violation. The basic idea behind the see-saw mechanism is to integrate out the heavy modes leading to higher-dimensional neutrino mass operators. Depending on the choice of the heavy particles one can classify variants of this mechanism, namely Type-I, -II, -III, Inverse, Double see-saw etc., both in supersymmetric (SUSY) and non-SUSY scenarios. Apart from neutrino masses and mixing, the deviation from the galactic rotation curve and bullet clusters provide concrete evidences in favor of dark matter (DM). Cosmological observations have also measured the relic density [2,3] of the DM with very high precision. But unfortunately the DM characteristics e.g. mass, spin and its nature i.e., cold, warm, single or multi component are yet to be determined.

All these shortcomings of the SM can be explained quite efficiently in SUSY. For example, the lightest supersymmetric particle (LSP) is an excellent cold DM candidate in the paradigm of the minimal supersymmetric standard model (MSSM) with $R$-parity 
conservation. On the other hand, MSSM with $R$-parity violation (RPV) is an intrinsic SUSY way to generate neutrino masses and mixing both at the tree level as well as at the one-loop [4] level. However, if $R$-parity is broken, the LSP becomes unstable and hence fails to explain the observed relic density of the universe. In such cases, the DM candidate could be a gravitino, axino, axion and keV sterile neutrino [5].

Concerning the experimental verification of proposed models, unfortunately, the early $13 \mathrm{TeV}$ run of the LHC has not found any signals $[6,7]$ in favor of SUSY. Although the non-observation of superpartners does not invalidate the idea of SUSY, it certainly questions the ability of MSSM to resolve the naturalness problem. So far, LHC has already ruled out gluinos lighter than $2 \mathrm{TeV}$ when the gluino and LSP masses are well separated. This in turn makes the whole colored sector heavy due to the logarithmic sensitivity to the ultra-violet (UV) scale through renormalisation group evolutions. Interestingly, this correlation is not generic and can be avoided within the models of $R$-symmetry and Dirac gauginos. One needs to extend the gauge sector of MSSM to $N=2$ representation to construct a Dirac gaugino mass. This require chiral superfields such as a singlet $\widehat{S}$, a triplet $\widehat{T}$ under $\mathrm{SU}(2)_{L}$ and an octet $\widehat{O}$ under $\mathrm{SU}(3)_{C}$, in the adjoint representation of the SM gauge group. These fields couple with bino, wino and gluinos respectively to generate Dirac masses for the gauginos. The presence of additional adjoint scalars cancel the UV logarithmic divergence for squark masses which results in a finite correction [8] only. Hence, the Dirac gluino masses can easily be made heavy. An immediate consequence of having heavy Dirac gluinos is the suppression of the gluino pair or squark-gluino associated pair production cross-sections due to kinematic suppression. However, it is important to note that gluino pair production proceeds through QCD, and the production cross-section for Dirac gluinos would be twice as large compared to the Majorana gluinos with equal masses [9]. This is based on the fact that Dirac gluinos have twice as many degrees of freedom than the Majorana gluinos. In addition, the pair production cross-section of squarks are also suppressed as it requires chirality flipping Majorana gluino masses in the propagator which is absent in these scenarios. This invariably weakens [10] the stringent bound on the first two generation squark masses. Also, trilinear scalar couplings ( $A$-terms) are absent in an $R$-symmetric framework and as a result flavor and $\mathrm{CP}$ violating interactions are suppressed [11].

Motivated by the issues pertaining to neutrino masses, DM and the non-observation of superpartners at the LHC, we propose a SUSY framework with an $R$-symmetry. Our prime aim in this paper is to generate neutrino masses within the $R$-symmetric Dirac gaugino framework. The $R$-charges are now identified with the lepton number of the SM fields. In general, we have the freedom to cast these charges and one can look into other assignments in [12-20]. An outcome of our $R$-charge assignment is the presence of bilinear and trilinear " $R$-parity violating" terms in the superpotential which are $R$ symmetric. When $R$-symmetry gets broken, the bilinear RPV terms in the superpotential generate bilinear RPV terms in the scalar potential. As a result, sneutrinos can acquire tiny vacuum expectation values (VEVs) proportional to the order parameter of $R$-breaking, i.e., gravitino mass. However, the superpotential and scalar potential have related sources of bilinear RPV. Therefore, such terms can be simultaneously rotated away. Nevertheless, the 


\begin{tabular}{|ccc|ccc|}
\hline Superfields & SM rep & $\mathrm{U}(1)_{R}$ & Superfields & SM rep & $\mathrm{U}(1)_{R}$ \\
\hline$\widehat{H}_{u}$ & $(1,2,1)$ & 0 & $\widehat{R}_{d}$ & $(1,2,-1)$ & 2 \\
$\widehat{H}_{d}$ & $(1,2,-1)$ & 0 & $\widehat{R}_{u}$ & $(1,2,1)$ & 2 \\
\hline$\widehat{Q}_{i}$ & $\left(3,2, \frac{1}{3}\right)$ & 1 & $\widehat{S}$ & $(1,1,0)$ & 0 \\
$\widehat{U}_{i}^{c}$ & $\left(\overline{3}, 1,-\frac{4}{3}\right)$ & 1 & $\widehat{T}$ & $(1,3,0)$ & 0 \\
$\widehat{D}_{i}^{c}$ & $\left(\overline{3}, 1, \frac{2}{3}\right)$ & 1 & $\widehat{\mathcal{O}}$ & $(8,1,0)$ & 0 \\
\hline$\widehat{L}_{i}$ & $(1,2,-1)$ & 2 & & & \\
$\widehat{E}_{i}^{c}$ & $(1,1,2)$ & 0 & & & \\
\hline
\end{tabular}

Table 1. The gauge quantum numbers under the $\mathrm{SM}$ gauge group $\mathrm{SU}(3)_{c} \otimes \mathrm{SU}(2)_{L} \otimes \mathrm{U}(1)_{Y}$ as well as the $\mathrm{U}(1)_{R}$ charge assignments of the chiral superfields residing in the model.

trilinear RPV terms will be present in the superpotential resulting in a mixing between light neutrinos and Dirac gauginos. Hence, one generates light neutrino masses at the tree-level. The RPV couplings will also allow the gravitino to decay to a neutrino and a photon. Such a gravitino is an excellent decaying dark matter candidate provided its lifetime is greater than the age of the universe and is consistent with diffuse gamma ray fluxes.

We categorize our paper in the following manner. We propose the model and discuss the basic features of our scenario in section 2. We emphasize on the specific choice of $R$-charges leading to the presence of bilinear and trilinear "RPV" terms in the superpotential. In section 2.2, we discuss soft SUSY breaking, $R$-symmetry breaking and also the generation of sneutrino VEVs. Before proceeding to the fermionic sector, we choose a particular basis to remove the redundancy of the RPV operators in this model. Physics should be independent of the choice of basis. Therefore, we define two basis independent parameters $\kappa$ and $\zeta$ through which bilinear RPV is manifested. In section 2.4, we show that within the paradigm of our construction, the bilinear RPV terms can be rotated away completely from the superpotential and the scalar potential. However, the trilinear terms will remain. In section 3, we explore the neutral fermion mass matrix. There we illustrate how successfully neutrino masses and mixing can be generated in a simplistic scenario through the trilinear superpotential couplings. While considering normal as well as inverted hierarchies, we obtain the constraints on the relevant superpotential parameters. In section 4 we explore the possibility of gravitino dark matter in our model satisfying necessary constraints. We conclude by providing a direction to explore this scenario in collider experiments in section 5 .

\section{The model}

Our scenario is based on a SUSY framework with an added global $R$-symmetry where the gauginos are Dirac type unlike the MSSM. The choice of $R$-charges is shown in table 1 . It is rather straightforward to show that the scalars share the same $R$-charge with their corresponding superfields whereas for fermions they are one less. Similarly, the gauginos have $R$-charge one and the corresponding gauge bosons have zero $R$-charge. From table 1 , 
we note that the lepton number of the SM particles can be identified with their $R$-charges. The lepton number of the superpartners can be non-standard. The definition of our $R$ charge assignment can also be understood as our choice of $R$ equals $R_{0}+L$ [14]. Here $R_{0}$ is the traditional $R$-charge assignment in MRSSM $[21-24]^{1}$ and $L$ stands for the lepton number. In addition, an invariant action demands the superpotential to have $R$-charge of two units. Hence, the allowed terms in superpotential are:

$$
\begin{aligned}
W_{\mathrm{MSSM}} & =y_{u}^{i j} \widehat{U}_{i}^{c} \widehat{Q}_{j} \widehat{H}_{u}-y_{d}^{i j} \widehat{D}_{i}^{c} \widehat{Q}_{j} \widehat{H}_{d}-y_{e}^{i j} \widehat{E}_{i}^{c} \widehat{L}_{j} \widehat{H}_{d}-y_{r}^{i} \widehat{E}_{i}^{c} \widehat{R}_{d} \widehat{H}_{d}, \\
W_{\mathrm{adj}} & =\lambda_{S}^{u} \widehat{S} \widehat{R}_{d} \widehat{H}_{u}-\lambda_{T}^{u} \widehat{H}_{u} \widehat{T} \widehat{R}_{d}+\lambda_{S}^{d} \widehat{S} \widehat{H}_{d} \widehat{R}_{u}-\lambda_{T}^{d} \widehat{R}_{u} \widehat{T} \widehat{H}_{d}, \\
W_{\text {"bi-RPV" }} & =\mu_{i} \widehat{H}_{u} \widehat{L}_{i}, \\
W_{\text {"tri-RPV" }} & =\xi_{i} \widehat{S} \widehat{L}_{i} \widehat{H}_{u}-\eta_{i} \widehat{H}_{u} \widehat{T} \widehat{L}_{i}, \\
W_{\mu} & =\mu_{u} \widehat{R}_{d} \widehat{H}_{u}+\mu_{d} \widehat{H}_{d} \widehat{R}_{u} .
\end{aligned}
$$

The total superpotential is then $W=W_{\text {MSSM }}+W_{\text {adj }}+W$ "bi-RPV" $+W$ "tri-RPV" $+W_{\mu}$. The triplet $\widehat{T}$ under $\mathrm{SU}(2)_{L}$ is parametrised as $\widehat{T}=\sum_{a=1,2,3} \widehat{T}^{(a)}$, where $\widehat{T}^{(a)}=T_{a} \sigma^{a} / 2$, $\sigma^{a}$ 's being the Pauli matrices. We denote the components of the triplet field as $T_{3}=T_{0}$, $T_{+}=\left(T_{1}-i T_{2}\right) / \sqrt{2}$ and $T_{-}=\left(T_{1}+i T_{2}\right) / \sqrt{2}$. In eq. $(2.1), \widehat{H}_{u}, \widehat{H}_{d}, \widehat{L}_{i}, \widehat{E}_{i}^{c}, \widehat{Q}_{i}, \widehat{U}_{i}^{c}, \widehat{D}_{i}^{c}$ are the usual MSSM fields. Further, $\lambda^{u}, \lambda^{d}, \xi_{i}, \eta_{i}, y_{u}, y_{d}, y_{e}$ are trilinear/Yukawa couplings and $\mu_{i}, \mu_{u}$ and $\mu_{d}$ are couplings with mass dimension one. The traditional higgsino mass term $(\mu)$ is forbidden in $R$-symmetric models. To generate higgsino-like chargino and neutralino masses, it is mandatory to include two additional $\mathrm{SU}(2)$-doublet chiral superfields $\widehat{R}_{u}$ and $\widehat{R}_{d}$ carrying non-zero $R$-charges. The presence of an $R$-charge for these two fields imply that $R$-symmetry cannot be spontaneously broken in the visible sector. Otherwise one has to encounter massless $R$-axions. These doublets are also known as inert doublets in the literature.

We like to stress that both the bilinear and trilinear "RPV" but $R$-symmetric terms are present in the superpotential due to the assignment of $R$-charges. Also $R$-symmetry prohibits baryonic "RPV" terms in the superpotential and in the process the stringent constraints from proton decays can be circumvented. Before discussing neutrino mass generation mechanism, we would like to first address soft SUSY breaking, $R$-symmetry breaking and the generation of sneutrino VEVs.

\subsection{Soft (super-soft) SUSY breaking interactions}

We choose to work in a scenario where SUSY (global) breaking is not associated with $R$-symmetry breaking. This can be achieved through both $D$ - and $F$-type spurions. For example, Dirac gaugino masses can be generated with the help of a spurion superfield $W_{\alpha}^{\prime}=\lambda_{\alpha}^{\prime}+\theta_{\alpha} D^{\prime}$ as $[25,26]$ :

$$
\mathcal{L}_{\text {Dirac }}^{\text {gaugino }}=\int d^{2} \theta \frac{W_{\alpha}^{\prime}}{\Lambda}\left[\kappa_{1} W_{1 \alpha} \widehat{S}+\kappa_{2} \operatorname{Tr}\left(W_{2 \alpha} \widehat{T}\right)+\kappa_{3} \operatorname{Tr}\left(W_{3 \alpha} \widehat{O}\right)\right]+\text { h.c. },
$$

where $W_{i \alpha}$ and $W_{i \alpha}^{\prime}$ have $R$-charge 1 , i.e., $R\left[\lambda_{i \alpha}\right]=R\left[\lambda_{i \alpha}^{\prime}\right]=1$ and $R\left[D^{\prime}\right]=0$. The integration over the Grassmann co-ordinates generates the Dirac gaugino masses $M_{i}^{D} \sim$

\footnotetext{
${ }^{1}$ We note in passing that $R_{0}$ is 1 for the lepton and quark superfields.
} 
$\kappa_{i}\left\langle D^{\prime}\right\rangle / \Lambda$. Here, $i=1,2,3$ represent masses for $\mathrm{U}(1)_{Y}, \mathrm{SU}(2)_{L}$ and $\mathrm{SU}(3)_{C}$ gauginos and $\Lambda$ refers to the messenger scale. After the discovery of Higgs boson with mass around $125 \mathrm{GeV}$, it is important to address the status of Higgs mass within the given scenario. In a purely supersoft scenario, the scalar masses are one-loop suppressed compared to the gaugino masses. Consequently, because of $D$-flatness of the scalar potential [8], the tree level quartic term for the Higgs field is vanishingly small. This is challenging from the perspective of fitting the Higgs mass around $125 \mathrm{GeV}$. Thus instead of working in the generalized supersoft supersymmetry framework [27], we consider $F$-type breaking [14, 16] also. In our model, the scalar masses can be generated through such $F$-type spurion defined as $\widehat{X}=x+\theta^{2} F_{X}[14]$ which allows the following $\mathrm{U}(1)_{R}$ preserving operators

$$
\begin{aligned}
& \int d^{4} \theta \frac{\widehat{X}^{\dagger} \widehat{X}}{\Lambda^{2}}\left[\sum_{i} \widehat{\Phi}_{i}^{\dagger} \widehat{\Phi}_{i}+\left\{\widehat{H}_{u} \widehat{H}_{d}+\epsilon \Lambda \widehat{S}+\widehat{S}^{2}+\widehat{T}^{2}+\left(\frac{1}{\Lambda} \times \text { cubic }\right)+\text { h.c. }\right\}\right] \\
& \int d^{2} \theta \frac{X}{\Lambda}\left(\widehat{S} \widehat{T}^{2}+\widehat{S} \widehat{O}^{2}+\widehat{S}^{3}\right)+\text { h.c., }
\end{aligned}
$$

and can automatically generate the following $\mathrm{U}(1)_{R}$ preserving renormalizable soft SUSY breaking terms

$$
\mathcal{L}_{\text {soft }}=\sum_{i} m_{i}^{2} \phi_{i}^{\dagger} \phi_{i}+\left[t_{S} S+\frac{1}{2} b_{S} S^{2}+B_{\mu} H_{u} H_{d}+\ldots\right]
$$

The soft mass squared terms are proportional to $\left\langle F_{X}\right\rangle^{2} / \Lambda^{2} \equiv M_{\text {SUSY }}^{2}$ where we consider same magnitude for the $F$ - and $D$-type VEVs. Such a mechanism also generates a scalar singlet tadpole $t_{S} S$. However, as long as $t_{S}<M_{\mathrm{SUSY}}^{3}$, such a tadpole is not expected to destabilize the hierarchy [28]. Nevertheless, due to the absence of $R$-breaking terms $B_{\mu_{i}} H_{u} \tilde{\ell}_{i}$ in the scalar sector, sneutrinos cannot acquire VEV. This is an important ingredient for neutrino mass generation in traditional bilinear RPV scenarios.

\section{$2.2 \quad R$-symmetry breaking}

It is well established that our universe is associated with a vanishingly small vacuum energy or cosmological constant. To explain this from the perspective of spontaneously broken supergravity theory in the hidden sector, the superpotential needs to acquire a non-zero VEV. Since the superpotential carries non-zero $R$-charge, therefore, $\langle W\rangle \neq 0$ implies breaking of $R$-symmetry. As a result, the gravitino would also acquire a mass which turns out to be the order parameter of $R$-symmetry breaking. The $R$-breaking information is then communicated to the visible sector through anomaly mediation and in the process the following $R$-symmetry breaking terms are generated

$$
\mathcal{L}_{\not{R}}=M_{1} \widetilde{b b}+M_{2} \widetilde{w} \widetilde{w}+M_{3} \widetilde{g} \widetilde{g}+A_{u} \widetilde{u}_{R} \widetilde{u}_{L}^{*} H_{u}^{0}+A_{d} \widetilde{d}_{R} \widetilde{d}_{L}^{*} H_{d}^{0}+A_{\ell} \widetilde{\ell}_{R} \widetilde{\ell}_{L}^{*} H_{d}^{0}+\text { h.c. }
$$

where the Majorana gaugino masses are generated through small $R$-breaking effects as

$$
M_{i}=\frac{g_{i}^{2}}{16 \pi^{2}} b_{i} m_{3 / 2} \quad(i=1,2,3)
$$


with beta functions

$$
b_{1}=33 / 5, \quad b_{2}=1, \quad b_{3}=-3 .
$$

The small $R$ symmetry-breaking trilinear scalar interactions are as follows

$$
\begin{aligned}
A_{\tau} & =\frac{m_{3 / 2}}{16 \pi^{2}}\left(-\frac{9}{5} g_{1}^{2}-3 g_{2}^{2}+3 Y_{b}^{2}+4 Y_{\tau}^{2}\right), \\
A_{t} & =\frac{m_{3 / 2}}{16 \pi^{2}}\left(-\frac{13}{15} g_{1}^{2}-3 g_{2}^{2}-\frac{16}{3} g_{3}^{2}+6 Y_{t}^{2}+Y_{b}^{2}\right), \\
A_{b} & =\frac{m_{3 / 2}}{16 \pi^{2}}\left(-\frac{7}{15} g_{1}^{2}-3 g_{2}^{2}-\frac{16}{3} g_{3}^{2}+Y_{t}^{2}+6 Y_{b}^{2}+Y_{\tau}^{2}\right) .
\end{aligned}
$$

It is also important to note that the presence of a conformal compensator field $\Sigma=$ $1+\theta^{2} m_{3 / 2}$ invariably generates a $B \mu_{i}$ term [29] in the superpotential through the following operator:

$$
\mathcal{L}=\int d^{2} \theta \Sigma^{3} \mu_{i} \widehat{H}_{u} \widehat{L}_{i}
$$

After scaling out this compensator field with $\widehat{\Phi}^{\prime}=\Sigma \widehat{\Phi}$ where $\widehat{\Phi}$ is a chiral superfield, we generate a bilinear term $\left(H_{u} \widetilde{\ell}_{i}\right)$ in the scalar potential

$$
B \mu_{i}=\mu_{i} m_{3 / 2}
$$

Hence, the $B \mu$ term is always aligned with the $\mu$ term. Such terms are $R$-breaking effects and proportional to the gravitino mass. The presence of this small effect would generate tiny sneutrino VEVs which might become important for neutrino mass-mixing as we discuss in the next section.

\subsection{Sneutrino VEV}

To compute the sneutrino VEVs, one has to include the contributions from $F$-, $D$ - and soft SUSY breaking terms. The additional pieces associated with $\mathrm{SU}(2)_{L}$ and $\mathrm{U}(1)_{Y}$ in the $D$-terms are

$$
D_{2}^{a}=g\left(H_{u}^{\dagger} \tau^{a} H_{u}+\widetilde{\ell}_{i}^{\dagger} \tau^{a} \widetilde{\ell}_{i}+T^{\dagger} \lambda^{a} T\right)+\sqrt{2}\left(M_{2}^{D} T^{a}+M_{2}^{D} T^{a^{\dagger}}\right),
$$

where $\tau^{a}$ and $\lambda^{a}$ 's represent the $\mathrm{SU}(2)$ generators in the fundamental and adjoint representations respectively. Similarly, the weak hyper-charge contribution $D_{Y}$ is given by

$$
D_{Y}=\frac{g^{\prime}}{2}\left(H_{u}^{\dagger} H_{u}-\widetilde{\ell}_{i}^{\dagger} \widetilde{\ell}_{i}\right)+\sqrt{2} M_{1}^{D}\left(S+S^{\dagger}\right) .
$$

The tree level scalar potential terms which participate in the sneutrino field minimization equations are

$$
\begin{aligned}
V_{F} & =\left|\mu_{i}\right|^{2}\left|\widetilde{\nu}_{i}^{0}\right|^{2}, \\
V_{\text {soft }} & =\widetilde{m}^{2}\left|\widetilde{\nu}_{i}^{0}\right|^{2}+B \mu_{i} H_{u}^{0} \widetilde{\nu}_{i}, \\
V_{D} & =\left[\frac{\left(g^{2}+g^{\prime 2}\right)}{8}\left|\widetilde{\nu}_{i}^{0}\right|^{2}-\sqrt{2} g^{\prime} M_{1}^{D} v_{S}+\sqrt{2} g M_{2}^{D} v_{T}\right]\left|\widetilde{\nu}_{i}^{0}\right|^{2} .
\end{aligned}
$$


In the limit $v_{S}, v_{T} \rightarrow 0$, the sneutrino VEVs can be well approximated as

$$
\left\langle\widetilde{\nu}_{i}\right\rangle \simeq-\frac{B \mu_{i} v_{u}}{\widetilde{m}_{i}^{2}+\mu_{i}^{2}}
$$

Such a choice of the singlet and triplet VEVs also ensures that these fields are very heavy through their respective minimization equations. Assuming $\left\langle H_{u}^{0}\right\rangle=v_{u} \sim \widetilde{m}_{i}$ i.e., at the electroweak scale, we find $\left\langle\widetilde{\nu}_{i}\right\rangle \sim B \mu_{i} / \widetilde{m} \sim \mu_{i} m_{3 / 2} / \widetilde{m}$. Off course, in the same manner the inert scalars $\left(R_{u}, R_{d}\right)$ would also acquire a VEV and as a result would mix with the Higgs fields. However, that mixing is also suppressed by the $R$-breaking parameter $m_{3 / 2}$ and does not play any important role in the phenomenological description.

\subsection{Choice of basis}

In the usual framework of bilinear RPV-MSSM [30-33], the lepton and the Higgs superfields are at the same footing [34] as they carry the same gauge charges. The lepton number violating couplings depend on the choice of $\left(\widehat{H}_{d}, \widehat{L}_{i}\right)$ basis. Thus it is important to explicitly mention the choice of basis in which the analysis is being performed. However, physics should not depend on such choices. Therefore, two basis independent parameters $\sin \kappa$ and $\sin \zeta$ are often introduced in the literature [35-38] which encapsulate the total lepton number violation in the superpotential as well as in the scalar potential respectively.

In our scenario, both the superfields $\widehat{R}_{d}$ and $\widehat{L}_{i}$ carry the same charges as can be seen from table 1 . Hence, in terms of the four-vector $\widehat{L}_{\alpha}, \alpha=0,1,2,3$ where $\widehat{L}_{0} \equiv \widehat{R}_{d}$, the renormalizable superpotential can be written as:

$$
\begin{aligned}
W= & y_{u}^{i j} \widehat{U}_{i}^{c} \widehat{Q}_{j} \widehat{H}_{u}-y_{d}^{i j} \widehat{D}_{i}^{c} \widehat{Q}_{j} \widehat{H}_{d}-y_{e}^{i \alpha} \widehat{E}_{i}^{c} \widehat{L}_{\alpha} \widehat{H}_{d}+\xi_{\alpha} \widehat{S} \widehat{L}_{\alpha} \widehat{H}_{u}-\eta_{\alpha} \widehat{H}_{u} \widehat{T} \cdot \widehat{L}_{\alpha}+\lambda_{s}^{d} \widehat{S} \widehat{H}_{d} \widehat{R}_{u} \\
& -\lambda_{T}^{d} \widehat{R}_{u} \widehat{T} \widehat{H}_{d}+\mu_{d} \widehat{H}_{d} \widehat{R}_{u}+\mu_{\alpha} \widehat{L}_{\alpha} \widehat{H}_{u} .
\end{aligned}
$$

Similarly, the scalar potential consisting of soft and super-soft terms reduces to the following form:

$$
\begin{aligned}
V_{\text {soft }}= & \sum_{i} m_{i}^{2} \phi_{i}^{\dagger} \phi_{i}+M_{1}^{D} \widetilde{b} \widetilde{S}+M_{2}^{D} \widetilde{W} \widetilde{T}+M_{3}^{D} \widetilde{g} \widetilde{O}+M_{1} \widetilde{b b}+M_{2} \widetilde{W} \widetilde{W}+M_{3} \widetilde{g} \widetilde{g}+B_{\alpha} H_{u} \widetilde{L}_{\alpha} \\
& +\left[t_{S} S+\frac{1}{2} b_{S} S^{2}+B_{\mu} H_{u} H_{d}+\ldots\right]+A_{u} \widetilde{u}_{R} \widetilde{u}_{L}^{*} H_{u}^{0}+A_{d} \widetilde{d}_{R} \widetilde{d}_{L}^{*} H_{d}^{0}+A_{l} \widetilde{l}_{R} \widetilde{l}_{L}^{*} H_{d}^{0} .
\end{aligned}
$$

In the zero-sneutrino VEV basis we define $\widehat{R}_{d}$ in terms of the $\widehat{L}_{\alpha}$ superfields as

$$
\widehat{R}_{d}=\frac{1}{v_{R}} \sum_{\alpha} v_{\alpha} \widehat{L}_{\alpha}
$$

where $v_{R} \equiv\left(\sum_{\alpha} v_{\alpha} v^{\alpha}\right)^{1 / 2}$ and gets generated due to $R$-symmetry breaking. Likewise, the four vector superfield $\widehat{L}_{\alpha}$ can now be defined in terms of the usual slepton superfields $\widehat{L}_{i}$ with vanishing VEVs and $\widehat{R}_{d}$ in the following way

$$
\widehat{L}_{\alpha}=\frac{v_{\alpha}}{v_{R}} \widehat{R}_{d}+\sum_{i} e_{\alpha i} \widehat{L}_{i}
$$


Even then, there is a freedom to rotate the lepton $\left(\widehat{L}_{i}\right)$ superfields arbitrarily. We choose that only a single lepton superfield couples to $\widehat{H}_{u}$ in the superpotential. This allows us to rewrite the superpotential in terms of basis independent quantities by plugging eqs. (2.17) and (2.18) in eq. (2.15) as

$$
\begin{aligned}
W= & y_{u}^{i j} \widehat{U}_{i}^{c} \widehat{Q}_{j} \widehat{H}_{u}-y_{d}^{i j} \widehat{D}_{i}^{c} \widehat{Q}_{j} \widehat{H}_{d}-\widetilde{y}_{e}^{i} \widehat{E}_{i}^{c} \widehat{R}_{d} \widehat{H}_{d}-\widetilde{y}_{e}^{i j} \widehat{E}_{i}^{c} \widehat{L}_{j} \widehat{H}_{d}+\widetilde{\lambda}_{S}^{u} \widetilde{S}_{R_{d}} \widetilde{H}_{u}+\widetilde{\xi}_{i} \widehat{S} \widehat{L}_{i} \widehat{H}_{u} \\
& -\widetilde{\lambda}_{T}^{u} \widehat{H}_{u} \widehat{T} \widehat{R}_{d}-\widetilde{\eta}_{i} \widehat{H}_{u} \widehat{T} \widehat{L}_{i}+\lambda_{S}^{d} \widehat{S} \widehat{H}_{d} \widehat{R}_{u}-\lambda_{T}^{d} \widehat{R}_{u} \widehat{T} \widehat{H}_{d}+\mu_{d} \widehat{H}_{d} \widehat{R}_{u} \\
& +\mu_{u} \cos \kappa \widehat{R}_{d} \widehat{H}_{u}+\mu_{u} \sin \kappa \widehat{L}_{3} \widehat{H}_{u}
\end{aligned}
$$

where

$$
\begin{array}{rlrl}
\widetilde{\xi}_{i} & =\sum_{\alpha} \xi^{\alpha} e_{\alpha i}, & \widetilde{\lambda}_{S}^{u}=\sum_{\alpha} \frac{v_{\alpha}}{v_{R}} \xi^{\alpha}, & \widetilde{y}_{e}^{i}=\sum_{\alpha} y_{e}^{\alpha i} \frac{v_{\alpha}}{v_{R}}, \\
\widetilde{\eta}_{i}=\sum_{\alpha} \eta^{\alpha} e_{\alpha i}, & \widetilde{\lambda}_{T}^{u}=\sum_{\alpha} \frac{v_{\alpha}}{v_{R}} \eta^{\alpha}, & \widetilde{y}_{e j}^{i}=\sum_{\alpha} y_{e}^{\alpha i} e_{\alpha j},
\end{array}
$$

and

$$
\cos \kappa \equiv \frac{1}{\mu_{u} v_{R}} \sum_{\alpha} \mu_{\alpha} v^{\alpha}
$$

with $\mu_{u} \equiv\left(\sum_{\alpha} \mu_{\alpha} \mu^{\alpha}\right)^{1 / 2}$ [4]. $\kappa$ is the angle between the four-vectors $\mu_{\alpha}$ and $v_{\alpha}$. It is evident from eq. (2.14) that $v_{\alpha} \| B_{\alpha}$, i.e., $v_{\alpha} \| \mu_{\alpha}$ and therefore, $\sin \kappa=0$. Though $\mu_{\alpha}$ and $v_{\alpha}$ are basis dependent quantities their relative angle $\kappa$ does not depend on the choice of basis for $\widehat{L}_{\alpha}$ superfields. As a result, the effect of bilinear RPV terms can be rotated away completely from the superpotential.

Similarly, the bilinear $R$-parity violation in the scalar sector can be parametrised in terms of the angle $\zeta$ formed by four-vectors $B_{\alpha}$ and $v_{\alpha}$ as:

$$
\cos \zeta \equiv \frac{1}{B v_{R}} \sum_{\alpha} B_{\alpha} v^{\alpha}
$$

where $B \equiv\left(\sum_{\alpha} B_{\alpha} B^{\alpha}\right)^{1 / 2}$. But it is clear from the earlier section that $B_{\alpha}, \mu_{\alpha}, v_{\alpha}$ are also aligned together. This implies $\sin \zeta=0$ and thus allows us to rotate away the bilinear terms from the scalar sector also. Therefore, in reality, the bilinear RPV terms do not play any role in generating neutrino masses and mixing. However, trilinear terms in the superpotential can not be rotated away simultaneously. These terms play crucial role for neutrino mass generation as discussed in the following section.

\section{The fermion sector}

In this section we will consider both neutral and charged fermion sectors. The mixing between neutral fermions and neutrinos lead to neutrino masses at the tree level. Similarly, chargino mixes with the charged leptons which may potentially give rise to lepton number violating processes. 


\subsection{The neutral fermion sector}

The Lagrangian corresponding to the neutral fermion sector after $R$-symmetry breaking contain the following terms

$$
\begin{aligned}
\mathcal{L}_{f^{0}}= & M_{1}^{D} \widetilde{b} \widetilde{S}+M_{2}^{D} \widetilde{w}^{0} \widetilde{T}+\mu_{u} \widetilde{H}_{u}^{0} \widetilde{R}_{d}^{0}+\mu_{d} \widetilde{H}_{d}^{0} R_{u}^{0}+\widetilde{\lambda}_{S}^{u} v_{u} \widetilde{S} \widetilde{R}_{d}^{0}+\widetilde{\lambda}_{S}^{d} v_{d} \widetilde{S} \widetilde{R}_{u}^{0}+\widetilde{\lambda}_{T}^{u} v_{u} \widetilde{T}^{0} \widetilde{R}_{d}^{0} \\
& +\widetilde{\lambda}_{T}^{d} v_{d} \widetilde{R}_{u}^{0} \widetilde{T}^{0}+M_{1} \widetilde{b b}+M_{2} \widetilde{w}^{0} \widetilde{w}^{0}+M_{S} \widetilde{S} \widetilde{S}+M_{T} \widetilde{T}^{0} \widetilde{T}^{0}+\frac{g^{\prime} v_{u}}{\sqrt{2}} \widetilde{b} \widetilde{H}_{u}^{0}-\frac{g^{\prime} v_{d}}{\sqrt{2}} \widetilde{b} \widetilde{H}_{d}^{0} \\
& -\frac{g v_{u}}{\sqrt{2}} \widetilde{w}^{0} \widetilde{H}_{u}^{0}+\frac{g v_{d}}{\sqrt{2}} \widetilde{w}^{0} \widetilde{H}_{d}^{0}+\widetilde{\xi}_{i} v_{u} \widetilde{S} \nu_{i}+\frac{\widetilde{\eta}_{i} v_{u}}{\sqrt{2}} \widetilde{T}^{0} \nu_{i}+\left(\widetilde{\xi}_{i} v_{S}+\widetilde{\eta}_{i} v_{T}\right) \widetilde{H}_{u}^{0} \nu_{i} .
\end{aligned}
$$

Here, " $i$ " stands for $e, \mu$ and $\tau$.

The Lagrangian mass terms expressed in the basis $f^{0} \equiv\left(\widetilde{b}, \widetilde{S}, \widetilde{w}^{0}, \widetilde{T}^{0}, \widetilde{H}_{u}^{0}, \widetilde{R}_{d}^{0}, \widetilde{H}_{d}^{0}, \widetilde{R}_{u}^{0}, \nu_{i}\right)$ can be written schematically as

$$
\mathcal{L}_{f^{0}}^{\operatorname{mass}}=\frac{1}{2}\left(f^{0}\right)^{T} M_{N} f^{0}
$$

where

$$
M_{N}=\left(\begin{array}{cc}
m_{\widetilde{f}^{0}} & m_{D} \\
m_{D}^{T} & 0
\end{array}\right),
$$

with

$$
m_{\widetilde{f}^{0}}=\left(\begin{array}{cccccccc}
M_{1} & M_{1}^{D} & 0 & 0 & \frac{g^{\prime} v_{u}}{\sqrt{2}} & 0 & -\frac{g^{\prime} v_{d}}{\sqrt{2}} & 0 \\
M_{1}^{D} & M_{S} & 0 & 0 & 0 & \widetilde{\lambda}_{S}^{u} v_{u} & 0 & \widetilde{\lambda}_{S}^{d} v_{d} \\
0 & 0 & M_{2} & M_{2}^{D} & -\frac{g v_{u}}{\sqrt{2}} & 0 & \frac{g v_{d}}{\sqrt{2}} & 0 \\
0 & 0 & M_{2}^{D} & M_{T} & 0 & \frac{\tilde{\lambda}_{T}^{u} v_{u}}{\sqrt{2}} & 0 & \frac{\widetilde{\lambda}_{T}^{d} v_{d}}{\sqrt{2}} \\
\frac{g^{\prime} v_{u}}{\sqrt{2}} & 0 & -\frac{g v_{u}}{\sqrt{2}} & 0 & 0 & \mu & 0 & 0 \\
0 & \widetilde{\lambda}_{S}^{u} v_{u} & 0 & \frac{\widetilde{\lambda}_{T}^{u} v_{u}}{\sqrt{2}} & \mu & 0 & 0 & 0 \\
-\frac{g^{\prime} v_{d}}{\sqrt{2}} & 0 & \frac{g v_{d}}{\sqrt{2}} & 0 & 0 & 0 & 0 & \mu \\
0 & \widetilde{\lambda}_{S}^{d} v_{d} & 0 & \frac{\tilde{\lambda}_{T}^{d} v_{d}}{\sqrt{2}} & 0 & 0 & \mu & 0
\end{array}\right),
$$

and

$$
m_{D}^{T}=\left(\begin{array}{cccccccc}
0 & \widetilde{\xi}_{1} v_{u} & 0 & \frac{\widetilde{\eta}_{1} v_{u}}{\sqrt{2}} & 0 & 0 & 0 & 0 \\
0 & \widetilde{\xi}_{2} v_{u} & 0 & \frac{\widetilde{\eta}_{2} v_{u}}{\sqrt{2}} & 0 & 0 & 0 & 0 \\
0 & \widetilde{\xi}_{3} v_{u} & 0 & \frac{\widetilde{\eta}_{3} v_{u}}{\sqrt{2}} & 0 & 0 & 0 & 0
\end{array}\right)
$$

Here, $\left\langle H_{u}^{0}\right\rangle=v_{u},\left\langle H_{d}^{0}\right\rangle=v_{d}$ with $v=\sqrt{v_{u}^{2}+v_{d}^{2}}$ and $\tan \beta=v_{u} / v_{d}$. We also consider $\mu_{u} \equiv \mu_{d} \equiv \mu$, and $M_{1} \sim M_{2} \sim M_{S} \sim M_{T} \sim m_{3 / 2} / 16 \pi^{2}$. For simplicity, we have chosen $v_{S}, v_{T} \sim 0$. We note, an order one value of the superpotential couplings $\widetilde{\lambda}_{S, T}^{u}$ provide substantial one-loop corrections to the up-type Higgs $[39,40]$ boson mass $m_{H_{u}}^{2}$. These corrections are 'stop-like' and an $125 \mathrm{GeV}$ Higgs boson can be obtained without requiring too heavy top squarks. Hence, we kept $\widetilde{\lambda}_{S, T}^{u}$ but assumed $\widetilde{\lambda}_{S, T}^{d}$ to be small. In the next section, we carry out a simplified analysis to explain neutrino masses and mixings. For brevity, we shall also make the following transformations: $\widetilde{\lambda}_{S}^{u} \sim \widetilde{\lambda}_{T}^{u} / \sqrt{2} \sim \lambda, \widetilde{\eta} / \sqrt{2} \rightarrow \widetilde{\eta}$. 


\subsection{Neutrino mass and mixing}

In order to obtain a quantitative estimation of the relevant parameters which satisfy neutrino masses and mixing, we choose all the $R$-symmetry preserving masses are of the same order, i.e., $M_{1}^{D} \sim M_{2}^{D} \sim \mu \sim v_{u} \equiv \widetilde{m}$. The structure of the effective neutrino mass matrix follows from the typical Type-I seesaw expression $\left[m_{D}^{T} m_{\widetilde{f}^{0}}^{-1} m_{D}\right]$ and represented as

$$
\left(m_{\nu}\right)_{i j} \simeq \frac{m_{3 / 2}}{16 \pi^{2}}\left[a \widetilde{\eta}_{i} \widetilde{\eta}_{j}+\frac{b}{2}\left(\widetilde{\eta}_{i} \widetilde{\xi}_{j}+\widetilde{\eta}_{j} \widetilde{\xi}_{i}\right)+c \widetilde{\xi}_{i} \widetilde{\xi}_{j}\right]
$$

where

$$
\begin{aligned}
& a=-\frac{\left[2-2 \sqrt{2} \lambda g^{\prime}+\left(g^{2}+g^{\prime 2}\right) \lambda^{2}\right]}{\left[2+\lambda\left(g-g^{\prime}\right)\left\{2 \sqrt{2}+\lambda\left(g-g^{\prime}\right)\right\}\right]}, \\
& b=-\frac{2\left[\sqrt{2} \lambda\left(g-g^{\prime}\right)+\left(g^{2}+g^{\prime 2}\right) \lambda^{2}\right]}{\left[2+\lambda\left(g-g^{\prime}\right)\left\{2 \sqrt{2}+\lambda\left(g-g^{\prime}\right)\right\}\right]}, \\
& c=-\frac{\left[2+2 \sqrt{2} \lambda g^{\prime}+\left(g^{2}+g^{\prime 2}\right) \lambda^{2}\right]}{\left[2+\lambda\left(g-g^{\prime}\right)\left\{2 \sqrt{2}+\lambda\left(g-g^{\prime}\right)\right\}\right]} .
\end{aligned}
$$

In principle, the parameters $\widetilde{\eta}$ and $\widetilde{\xi}$ can be varied within the validated range to fit the observed neutrino masses and mixing as showed in [41] for bilinear RPV scenario in MSSM. However, in that framework such a form of the neutrino mass matrix arises only after taking the loop corrections into account. Here we adopt a rather simplified approach [42] to estimate the values of these superpotential parameters such that neutrino masses and mixing can be successfully generated. The co-efficient of $\widetilde{\eta}_{i} \widetilde{\xi}_{j}$ vanishes for distinct values of $\lambda$ which can be obtained by setting $b=0$ in eq. (3.7). Under such approximation, the neutrino mass expression in eq. (3.6) turns out to be:

$$
\begin{aligned}
&\left.\left(m_{\nu}\right)_{i j}\right|_{\lambda=0} \simeq \frac{m_{3 / 2}}{16 \pi^{2}}\left[\widetilde{\eta}_{i} \widetilde{\eta}_{j}+\widetilde{\xi}_{i} \widetilde{\xi}_{j}\right] \\
&\left.\left(m_{\nu}\right)_{i j}\right|_{\lambda=-\frac{\sqrt{2}\left(g-g^{\prime}\right)}{\left(g^{2}+g^{\prime 2}\right)}} \simeq \frac{m_{3 / 2}}{16 \pi^{2}}\left[\frac{\left(1+\tan ^{2} \theta_{W}\right)}{2} \widetilde{\eta}_{i} \widetilde{\eta}_{j}+\frac{\left(1+\cot ^{2} \theta_{W}\right)}{2} \widetilde{\xi}_{i} \widetilde{\xi}_{j}\right] .
\end{aligned}
$$

Further assuming $\widetilde{\eta}_{i}>\widetilde{\xi}_{j}(\forall i, j)$, we can decompose the full neutrino mass matrix as

$$
\left(m_{\nu}\right)_{i j} \simeq \frac{m_{3 / 2}}{16 \pi^{2}}[\underbrace{\left(\begin{array}{ccccc}
\widetilde{\eta}_{1}^{2} & \widetilde{\eta}_{1} \widetilde{\eta}_{2} & \widetilde{\eta}_{1} \widetilde{\eta}_{3} \\
\widetilde{\eta}_{1} \widetilde{\eta}_{2} & \widetilde{\eta}_{2}^{2} & \widetilde{\eta}_{2} \widetilde{\eta}_{3} \\
\widetilde{\eta}_{1} \widetilde{\eta}_{3} & \widetilde{\eta}_{2} \widetilde{\eta}_{3} & \widetilde{\eta}_{3}^{2}
\end{array}\right)}_{\text {leading order }}+c_{1} \underbrace{\left(\begin{array}{ccccc}
\widetilde{\xi}_{1}^{2} & \widetilde{\xi}_{1} \widetilde{\xi}_{2} & \widetilde{\xi}_{1} \widetilde{\xi}_{3} \\
\widetilde{\xi}_{1} \widetilde{\xi}_{2} & \widetilde{\xi}_{2}^{2} & \widetilde{\xi}_{2} & \widetilde{\xi}_{3} \\
\widetilde{\xi}_{1} \widetilde{\xi}_{3} & \widetilde{\xi}_{2} \widetilde{\xi}_{3} & \widetilde{\xi}_{3}^{2}
\end{array}\right)}_{\text {perturbation }}] .
$$

where $c_{0} \equiv\left(1+\tan ^{2} \theta_{W}\right) / 2$ and $c_{1} \equiv\left(1+\cot ^{2} \theta_{W}\right) / 2$ for the second case of eq. (3.8). This mass matrix consists of leading $\sim \mathcal{O}\left(\tilde{\eta}_{i} \tilde{\eta}_{j}\right)$ and sub-leading, i.e., perturbation $\sim \mathcal{O}\left(\tilde{\xi}_{i} \tilde{\xi}_{j}\right)$ terms. The leading order matrix can generate only one massive light neutrino at the tree level as it is of rank one. Using the projective nature of the neutrino mass matrix one can 


\begin{tabular}{|c|cc|}
\hline Parameters & Normal ordering & Inverted ordering \\
\hline $\sin ^{2} \theta_{12}$ & $0.306_{-0.012}^{+0.012}$ & $0.306_{-0.012}^{+0.012}$ \\
\hline $\sin ^{2} \theta_{23}$ & $0.441_{-0.021}^{+0.027}$ & $0.587_{-0.024}^{+0.020}$ \\
\hline $\sin ^{2} \theta_{13}$ & $0.0216_{-0.00075}^{+0.00075}$ & $0.0218_{-0.00076}^{+0.00076}$ \\
\hline$\frac{\Delta m_{21}^{2}}{10^{-5} \mathrm{eV}^{2}}$ & $7.50_{-0.17}^{+0.19}$ & $7.50_{-0.17}^{+0.19}$ \\
\hline$\frac{\Delta m_{3 \ell}^{2}}{10^{-3} \mathrm{eV}^{2}}$ & $2.524_{-0.040}^{+0.039}$ & $-2.514_{-0.041}^{+0.038}$ \\
\hline
\end{tabular}

Table 2. Latest values of the neutrino oscillation parameters within $3 \sigma$ [1] error range. Note that, $\ell=1,2$ for normal and inverted hierarchies respectively.

rotate away one of the three mixing angles $\left(\theta_{12}\right)$ and finally the non-zero eigenvalue and the two non-zero mixing angles turn out to be

$$
\left[m_{\nu}\right]_{3} \simeq \frac{m_{3 / 2}}{16 \pi^{2}} c_{0}|\overrightarrow{\vec{\eta}}|^{2}, \quad \tan \theta_{13} \simeq-\frac{\widetilde{\eta}_{1}}{\left(\widetilde{\eta}_{2}^{2}+\widetilde{\eta}_{3}^{2}\right)^{1 / 2}}, \quad \tan \theta_{23} \simeq \frac{\widetilde{\eta}_{2}}{\widetilde{\eta}_{3}} .
$$

Here, the heaviest neutrino mass generated from the leading order terms of the effective neutrino mass matrix is denoted as $\left[m_{\nu}\right]_{3}$. These two mixing angles constrain the trilinear parameters $\widetilde{\eta}$. We now rewrite eq. (3.9) in a compact form as

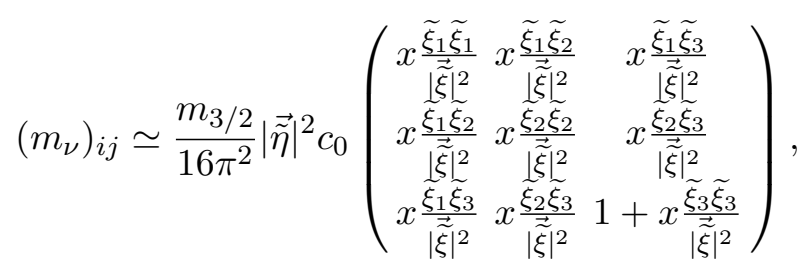

where $x=c_{1}|\overrightarrow{\tilde{\xi}}|^{2} / c_{0}|\overrightarrow{\vec{\eta}}|^{2}$. Under the assumption $\xi_{3} \sim 0$, the heaviest neutrino mass remains almost unaltered, and the other masses and the third mixing angle get generated as

$$
\left[m_{\nu}\right]_{1}=0, \quad\left[m_{\nu}\right]_{2} \simeq \frac{m_{3 / 2}}{16 \pi^{2}} c_{1}\left(\widetilde{\xi}_{1}^{2}+\widetilde{\xi}_{2}^{2}\right), \quad \tan \theta_{12} \simeq \frac{\widetilde{\xi}_{1}}{\widetilde{\xi}_{2}} .
$$

The recent data for the neutrino mass and mixing angles are shown in table 2 [1]. Using these fitted parameters we find the following constraints for normal (NH) and inverted (IH) hierarchies respectively

$$
\begin{aligned}
m_{3 / 2} \widetilde{\eta}_{3}^{2} & \simeq 6.5 \times 10^{-3} \mathrm{keV}(\mathrm{NH}), & m_{3 / 2} \widetilde{\eta}_{3}^{2} & \simeq 7.1 \times 10^{-3} \mathrm{keV}(\mathrm{IH}), \\
m_{3 / 2} \widetilde{\xi}_{2}^{2} & \simeq 4.5 \times 10^{-4} \mathrm{keV}(\mathrm{NH}), & m_{3 / 2} \widetilde{\xi}_{2}^{2} & \simeq 2.6 \times 10^{-3} \mathrm{keV}(\mathrm{IH}), \\
\widetilde{\eta}_{1} & \simeq-0.2 \widetilde{\eta}_{3}(\mathrm{NH}), & \widetilde{\eta}_{1} & \simeq-0.2 \widetilde{\eta}_{3}(\mathrm{IH}), \\
\widetilde{\eta}_{2} & \simeq 0.89 \widetilde{\eta}_{3}(\mathrm{NH}), & \widetilde{\eta}_{2} & \simeq 1.19 \widetilde{\eta}_{3}(\mathrm{IH}), \\
\widetilde{\xi}_{1} & \simeq 0.67 \widetilde{\xi}_{2}(\mathrm{NH}), & \widetilde{\xi}_{1} & \simeq 0.67 \widetilde{\xi}_{2}(\mathrm{IH}) .
\end{aligned}
$$

Moreover, a legitimate bound on the gravitino mass can be obtained when the DM constraints are taken into consideration. For example, for a $\mathrm{MeV}$ scale gravitino, $\widetilde{\eta} \sim 10^{-3}$ and $\widetilde{\xi} \sim 10^{-4}$ are the ball-park numbers which satisfy neutrino mass constraints. 
Before proceeding further, we would like to briefly sketch the distinct features of our methodology compared to that described in earlier papers. In refs. [12, 13], the idea of generating neutrino masses and mixing was proposed in a framework with leptonic $R$ symmetry and pseudo-Dirac gauginos. Two separate cases of $R$-symmetry breaking were considered namely anomaly mediation (AMRB) and Planck mediation (PMRB). In the AMRB scenario, it was noted that the neutrinos remain massless at the tree-level and only become massive after radiative corrections. This is noticeably different from our case. In our paper, we confine ourselves in the AMRB scenario as the Planck mediated $R$-breaking operators can be sequestered away. Unlike [13], neutrino masses and mixings can be explained at the tree level itself because of our specific $R$-charge assignments and the presence of trilinear terms $\left(\xi_{i} \widehat{S} \widehat{H}_{u} \widehat{L}_{i}\right.$ and $\left.\eta_{i} \widehat{H}_{u} \cdot \widehat{T} \cdot \widehat{L}_{i}\right)$. In addition, the $R$-charge of the $\widehat{L}$ superfield was considered to be zero in [13] which allowed sneutrinos to acquire large VEVs. Such VEVs were also not constrained by the Majorana masses of the neutrinos. Hence, sneutrinos could also play the role of a down-type Higgs field. Following our charge assignment, sneutrino VEVs can be rotated away completely. Hence, noticeably different phenomenology and signatures at the collider are obtained. Moreover, in ref. [13] a tentative bound on the gravitino mass of $m_{3 / 2}<0.5 \mathrm{GeV}$ was obtained from the study of neutrino masses. However, such a constrain is not be applicable in our scenario as the neutrino mass is proportional to $m_{3 / 2} \xi^{2}\left(m_{3 / 2} \eta^{2}\right) / 16 \pi^{2}$. The superpotential couplings $\xi, \eta$ give an additional handle to fit neutrino masses and hence the strict bound on the gravitino mass can be somewhat ameliorated. Further, the bound on the gravitino mass in our case comes from DM constraints, which in turn gives an estimate on the model parameters.

\subsection{Charged fermion sector}

The presence of trilinear terms $\left(\eta_{i} \widehat{L}_{i} \widehat{T} \widehat{H}_{u}\right)$ in the superpotential results in a mixing between charged leptons and charginos. As the sneutrino VEVs are rotated away, therefore the gauge couplings between the charged leptons and bino/wino vanish. However, the mixing between higgsino like charginos and charged leptons is present. Such a mixing may lead to lepton flavor violating (LFV) processes. Therefore, a robust bound on the relevant superpotential parameters can be obtained from LFV studies. The chargino mass matrix in the basis $\left(e_{L}, \mu_{L}, \tau_{L}, \widetilde{W}^{-}, \widetilde{T}^{-}, \widetilde{R}_{d}^{-}, \widetilde{H}_{d}^{-}\right)$and $\left(e_{R}, \mu_{R}, \tau_{R}, \widetilde{W}^{+}, \widetilde{T}^{+}, \widetilde{R}_{u}^{+}, \widetilde{H}_{u}^{+}\right)$can be written as:

$$
m_{\widetilde{f}^{+}}=\left(\begin{array}{ccccccc}
v_{d} y_{11} & v_{d} y_{21} & v_{d} y_{13} & 0 & \frac{v_{u} \widetilde{\eta}_{1}}{\sqrt{2}} & 0 & v_{S} \widetilde{\xi}_{1}-v_{T} \widetilde{\eta}_{1} \\
v_{d} y_{12} & v_{d} y_{22} & v_{d} y_{23} & 0 & \frac{v_{u} \tilde{\eta}_{2}}{\sqrt{2}} & 0 & v_{S} \widetilde{\xi}_{2}-v_{T} \widetilde{\eta}_{2} \\
v_{d} y_{13} & v_{d} y_{23} & v_{d} y_{33} & 0 & \frac{v_{u} \tilde{\eta}_{3}}{\sqrt{2}} & 0 & v_{S} \widetilde{\xi}_{3}-v_{T} \widetilde{\eta}_{3} \\
0 & 0 & 0 & M_{2} & M_{2}^{D}-g v_{T} & 0 & g v_{u} \\
0 & 0 & 0 & M_{2}^{D}+g v_{T} & M_{T} & -\lambda_{T}^{d} v_{d} & 0 \\
0 & 0 & 0 & 0 & \lambda_{T}^{u} v_{u} & 0 & -\mu_{u} \\
0 & 0 & 0 & g v_{d} & 0 & -\mu_{u} & 0
\end{array}\right) .
$$

We note that for $\widetilde{\eta} \sim 10^{-3}$, the mixing elements and the masses of the charged leptons remain unaltered. The constraint appearing from lepton flavor violation $[43,44]$ can be translated into the following bound $\left(v^{2} / M_{D}^{2}\right) \widetilde{\eta}^{2}<10^{-5} \Longrightarrow \widetilde{\eta}<5 \times 10^{-3}$. 


\section{Gravitino as dark matter}

In order to check the viability of gravitino DM, we first discuss the production of gravitino and then its possible decay modes. The lifetime of the gravitino should be more than the age of the universe for it to become a feasible DM candidate. In addition, the decaying DM has to be consistent with constraints from diffuse gamma rays also.

\subsection{Production of gravitinos}

To start with, we consider the evolution of the universe dictated by the standard model of cosmology. This assumes the presence of an inflationary phase after the Big-bang. Any trace of pre-inflationary matter or radiation gets diluted because of the expansion and super cooling of the universe. The inflationary phase continues till the inflaton field reaches the minima of the scalar potential.

The total amount of energy stored in the inflaton then gets transformed into relativistic matter leading to drastic rise in the temperature and entropy of the universe. As a result, the universe reaches its maximum temperature known as the reheating temperature $T_{R}$. The gravitino can then reach thermal equilibrium with its environment in the postreheating period. Although the number density of gravitino was negligible to start with, it gets generated through scattering and decays of particles (squarks and gluinos) which are in thermal equilibrium within the plasma. Assuming $m_{\mathrm{SUSY}} \ll T_{R}$ (at the computational level), the thermal relic density of the gravitino $[45,46]$ can be estimated as

$$
\Omega_{3 / 2} h^{2} \sim 0.1\left(\frac{1 \mathrm{GeV}}{m_{3 / 2}}\right)\left(\frac{T_{R}}{10^{7} \mathrm{GeV}}\right)\left(\frac{m_{\widetilde{g}}}{2 \mathrm{TeV}}\right)^{2}
$$

where $\Omega_{3 / 2} h^{2} \sim 0.1199$ [2]. The gravitino exchanges energy and momentum with the particles already present in the thermal bath. This leads to a state of maximum entropy in which the distribution function follows Fermi-Dirac or Bose-Einstein statistics $f(p)=\left[\exp \left(\frac{E(p)-\mu}{T}\right) \pm 1\right]^{-1}$, where $\mu$ is the chemical potential and ' + ' (-) sign stands for fermions (bosons). The thermal production of gravitinos would require $T_{R} \gg m_{\widetilde{g}} \sim 2 \mathrm{TeV}{ }^{2}$ otherwise the production of gravitinos would be exponentially (Boltzmann) suppressed as: $\exp (-m / T)[47]$. Even though this outcome heavily depends on the exact SUSY spectrum, the constraint on the reheating temperature can be mostly satisfied with

$$
m_{3 / 2} \gtrsim 200 \mathrm{keV} \text {. }
$$

Such a gravitino would constitute a cold DM [48]. Gravitino 'freeze-in' can also play an important role in the fast decay of the superpartners in thermal equilibrium induced by the short lifetime of gluinos, squarks and sleptons. In such scenarios the gravitino DM may suffer from an over-abundance problem. The only way to circumvent this issue would require lowering the reheating temperature below the SUSY scale [49]. But such a low

\footnotetext{
${ }^{2}$ In our scenario, gluinos are very heavy compared to the electro-weak gauginos. Thus a pair of gluinos may decay to $q \bar{q} \widetilde{\chi}_{1}^{0}$, where $q$ 's are the first two generation quarks. Based on this type of decay, LHC provides stringent constraints on the gluino mass $\geq 2 \mathrm{TeV}[6,7]$.
} 
reheating temperature $\left(T_{R} \sim 2 \mathrm{TeV}\right)$ could be troublesome [50] from the perspective of thermal leptogenesis where typical values of the reheating temperature is required to be around $\sim 10^{8}-10^{9} \mathrm{GeV}$.

\subsection{Gravitino decay width and life-time}

As mentioned earlier, gravitinos are metastable in our framework with typically large lifetime. The reason is two fold: first the couplings are suppressed by the supersymmetry breaking scale and second the smallness of the superpotential couplings $\eta$ and $\xi$. In general a small photino-neutrino mixing allows the gravitino to decay into a photon and a neutrino. At tree level this decay channel is prohibited in our case as sneutrino VEVs are rotated away. However, for small gravitino mass this decay is feasible at one-loop and turns out to be the dominating one over the three body decay of gravitino into fermions. Thus we can safely ignore the consequences of the latter decay mode and assume that our DM candidate, i.e., gravitino decays into a photon and a neutrino producing two monochromatic lines at energy exactly equal to $m_{\mathrm{DM}} / 2$. This decay width [51] of gravitino is well approximated by

$$
\Gamma\left(\widetilde{G} \rightarrow \nu_{i} \gamma\right) \simeq \frac{1}{32 \pi}\left|U_{\gamma \nu}\right|^{2} \frac{m_{3 / 2}^{3}}{M_{P}^{2}},
$$

where $\left|U_{\gamma \nu}\right|^{2}=\sum_{a=i+4}\left|\cos \theta_{W} Z_{a 1}+\sin \theta_{W} Z_{a 2}\right|^{2}$ and $M_{P} \sim 2.4 \times 10^{18} \mathrm{GeV}$ is the reduced Planck mass. We approximate $\left|U_{\gamma \nu}\right|^{2} \simeq \sum_{a=i+4}\left|N_{a 1}\right|^{2}$ where $m=1,2,3$. The gravitino decay width can be further simplified in terms of the model parameters as

$$
\Gamma\left(\widetilde{G} \rightarrow \nu_{i} \gamma\right) \simeq \frac{3}{32 \pi}\left[\frac{\xi_{i} v_{u}}{M_{D}}\right]^{2} \frac{m_{3 / 2}^{3}}{M_{P}^{2}} \cos ^{2} \theta_{W} .
$$

Consequently, the lifetime of the gravitino turns out to be

$$
\tau_{3 / 2} \simeq \frac{32 \pi}{3 \cos ^{2} \theta_{W}}\left(\frac{M_{D}}{\widetilde{\xi}_{i} v_{u}}\right)^{2} \frac{M_{P}^{2}}{m_{3 / 2}^{3}} \mathrm{GeV}^{-1} .
$$

In order for the gravitino to become a valid DM candidate, the first priority is its lifetime should be greater than the age of the universe, which is around $4.32 \times 10^{17} \mathrm{sec}$. From eq. (4.5) it is straightforward to find that for $\widetilde{\xi} \sim 10^{-3}$

$$
m_{3 / 2} \lesssim 10 \mathrm{GeV}
$$

\subsection{Photon flux from gravitino decays}

To circumvent the stringent constraints from the diffuse gamma ray sources, the lifetime of the DM needs to be much greater [52] than the age of the Universe ( $\gtrsim 10^{24}-10^{28}$ sec.). The neutrino flux however is vanquished by the atmospheric neutrino background in the energy range of few $\mathrm{MeV}$ to $\mathrm{GeV}$ which makes its detection more difficult compared to the gamma ray flux. In general one can think of two typical sources for a diffuse gamma ray background. Firstly, due to DM decay in the nearby Milky way halo and secondly DM decay at cosmological distances. For inner galaxy constraints, data from INTEGRAL [53] 


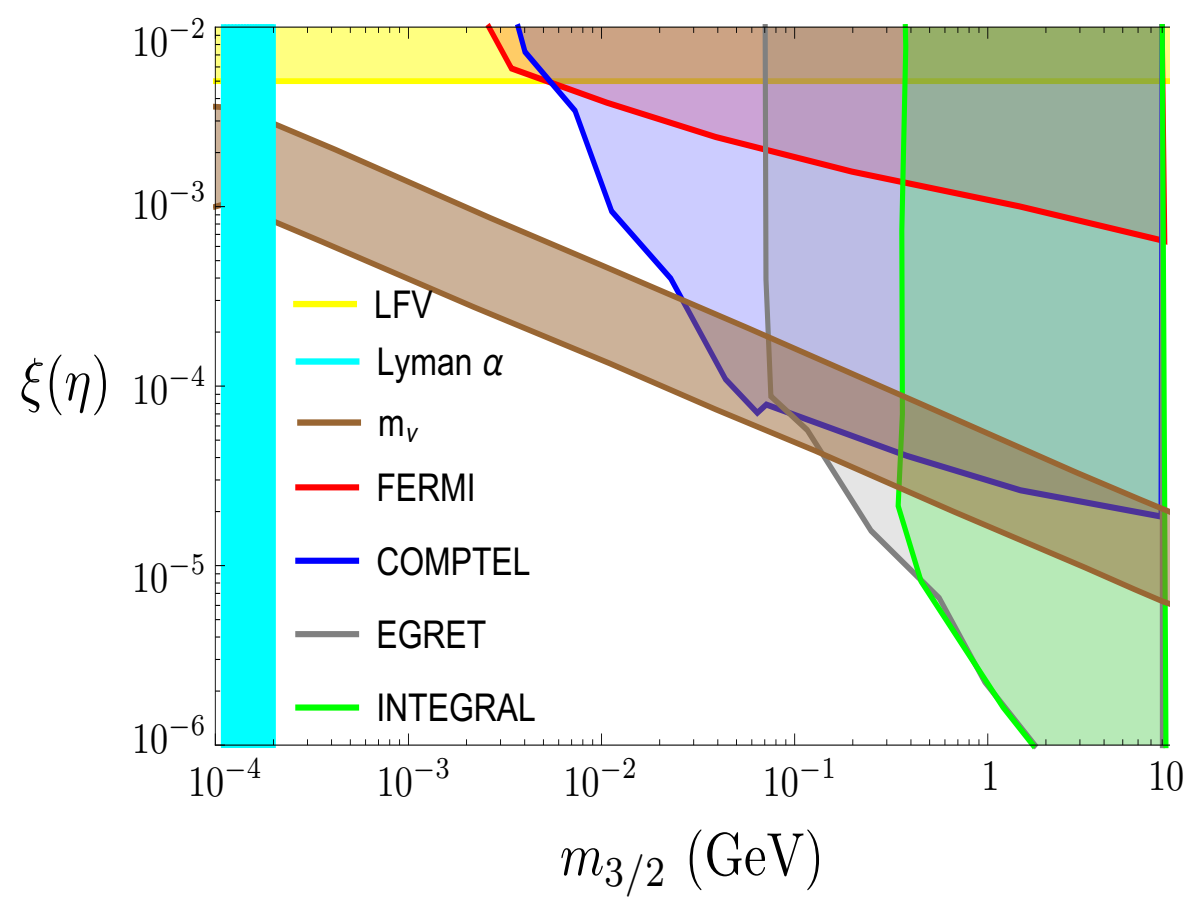

Figure 1. We show the allowed parameter space of the model. The photon fluxes considered from FERMI (red), COMPTEL (blue), EGRET (gray), INTEGRAL (green) experiments are translated to the $\xi(\eta)-m_{3 / 2}$ plane. Constraints from the Lyman- $\alpha$ forest experiment rules out gravitino mass less than $200 \mathrm{keV}$. We also overlay the neutrino mass constraints from eq. (3.13) where the brown shaded region satisfies constraints from neutrino mass data. The yellow shaded region is ruled out from the lepton flavor violating decays.

or COMPTEL [54] are used to probe photons within the mass range of $20 \mathrm{keV}-2 \mathrm{MeV}$. While the EGRET [55] and FERMI [56] experiments probe a region of $20 \mathrm{MeV}-10 \mathrm{GeV}$ and $200 \mathrm{MeV}-10 \mathrm{GeV}$ diffuse photons respectively. In most of the cases Navarro-FrenkWhite (NFW) [57, 58] profile of DM density is used. The bounds on the energy of the photons are quite sensitive to the DM density profile and may vary up to $\mathcal{O}(20 \%)$.

In figure 1, we show the allowed parameter space in the $m_{3 / 2}-\xi(\eta)$ plane compatible with the existing experimental constraints. The photon fluxes are taken from FERMI (red), COMPTEL (blue), EGRET (gray), INTEGRAL (green) experiments. Noticeably, Lyman$\alpha$ forest experiment rules out gravitino of mass less than $200 \mathrm{keV}$. We overlay the neutrino mass constraints depicted by the brown-shaded allowed region, obtained from eq. (3.13). The yellow region is disfavored from lepton flavor violating decays. From figure 1, it is clear that the allowed range for gravitino mass lies in between

$$
200 \mathrm{keV} \lesssim m_{3 / 2} \lesssim 0.1 \mathrm{GeV}
$$

Notice, the more robust upper bound on the gravitino mass comes from Lyman- $\alpha$ forest experiment. This limit coincides with the bound assuming the reheating temperature above the scale of superpartner masses. The lower limit on gravitino mass is achieved using the constraint from diffuse gamma ray fluxes in conjunction with neutrino oscillation 
data. Using eq. (4.1) for $m_{\widetilde{g}} \equiv 2 \mathrm{TeV}$, we can obtain a corresponding bound on the reheating temperature

$$
2 \times 10^{3} \mathrm{GeV} \lesssim T_{R} \lesssim 10^{6} \mathrm{GeV} .
$$

In this regime, electroweak baryogenesis [59-62] can explain the baryon asymmetry of the universe as the reheating temperature is well above the electroweak phase transition temperature. Also, in presence of R-symmetry, there are no $A$-terms in the scalar potential. This may allow the presence of large CP-violation originating from complex mass terms (Dirac gaugino masses, $\mu_{u / d}$ etc.) without affecting flavor, electric dipole moment and other low energy constraints $[11,63,64]$. This large CP-violation may turn out to be a suitable source of CP-asymmetry which can be translated into baryon asymmetry [11] through sphaleron effects. Another viable option for baryogenesis could be the Affleck-Dine mechanism [65]. During or after the reheating of the universe, the scalar superpartners carrying baryon or lepton numbers would decay into the SM fermions. The net baryon number carried by the SM fermions then may explain the observed excess of baryons over anti-baryons.

We would also like to point out that the viability of gravitino $\mathrm{DM}$ in $\mathrm{U}(1)_{R}$ models have already been discussed in [12]. In that framework, the alternative assignment of $R$-charges and large sneutrino VEVs ensure the tree level decay of the gravitino into a photon and a neutrino to be the most prominent. Such a scenario is severely constrained from diffuse gamma ray searches and can be tackled with much diluted gravitino density [12]. This can be achieved by assuming the reheating temperature to be lower than the SUSY scale. As a result, gravitino cannot explain the observed relic abundance of the universe. In our case, however, the absence of such tree level decay mode makes the gravitino a viable DM candidate and also the reheating temperature can be relatively larger compared to that in [12].

\section{Collider phenomenology}

In our model, gravitino turns out to be the LSP. With suitable choice of parameters, we can choose a valid SUSY spectrum with lightest neutralino to be the next-to-minimal supersymmetric particle (NLSP). Thus we would be left with a scenario where all the supersymmetric particles decay to the lightest neutralino which decays to a gravitino accompanied by either photon / $Z$-boson / Higgs. Such interactions are suppressed by the Planck scale and the resulting decay width will be very small, i.e., corresponding lifetime would be too large for the decay to occur within the collider. In addition, the NLSP also undergoes $R$-parity violating decay modes primarily in the following channel

$$
\widetilde{\chi}_{1,2}^{0} \rightarrow h \nu_{i}, \quad \gamma \nu_{i}
$$

The dominant decay width is noted down as [66]

$$
\Gamma\left(\widetilde{\chi}_{i}^{0} \rightarrow h \nu_{m}\right)=\frac{\alpha m_{\widetilde{\chi}_{i}^{0}}}{16 \sin ^{2} \theta_{W}}\left|\frac{\widetilde{\xi}_{m} \cos \alpha}{\sqrt{2}} N_{i 3} N_{11}\right|^{2}\left[1-\frac{m_{h}^{2}}{m_{\widetilde{\chi}_{i}^{0}}^{2}}\right]^{2} .
$$

Here we assume that $\widetilde{\chi}_{1,2}^{0}$ is either bino or singlino type. Notice that the same final state topology would arise if the neutralino is wino or triplino type. In that case the relevant 
parameter would be $\widetilde{\eta}$ instead of $\widetilde{\xi}$. This particular decay mode of the light neutralino to a neutrino and a Higgs boson gives rise to an interesting di-Higgs signature at the colliders [67]. Moreover, a pair produced charginos can also decay to pair of Higgs boson associated with opposite sign charged leptons through the $\widetilde{\eta}$ coupling. This is a distinct feature of our scenario. For our case, the typical values of $\widetilde{\xi}$ and $\widetilde{\eta}$ are around $\mathcal{O}\left(10^{-3}\right)$. This leads to rather prompt decays at the colliders. This is also different from the standard RPV case where characteristic signals come from longer decay lengths and displaced vertices due to the smallness of the sneutrino VEVs. In the wino-higgsino decoupled scenario the lightest neutralino is primarily an admixture of the bino-singlino states. In addition to the $h \nu$ final state, $Z \nu$ and $W^{ \pm} \ell^{\mp}$ decay modes are also present. One can easily have light higgsinos in this framework without much modifications in the neutrino sector and would also have interesting collider implications [68].

In generic bilinear RPV models, the mixing between the Higgs and the sneutrino induces a slight mass splitting between sneutrinos and anti-sneutrinos which gives rise to sneutrino oscillation [69] signatures. This mixing is controlled by the $B_{\alpha} H_{u} \widetilde{L}_{i}$ term in the scalar potential. Also the sneutrino VEVs generate trilinear couplings involving lepton (quark) and slepton (squark) fields which are interesting channels to look for [70]. However, such signals are not probable in our case as the bilinear terms are rotated away completely. Moreover, the presence of small $R$-breaking effects would create a slight mass splitting between the pair of Dirac neutralinos. These pseudo-Dirac neutralinos can give rise to neutralino oscillation [71] signatures at the colliders. In typical cases, these neutralinos can decay to a $h \widetilde{G}$. The difference between the distribution of the displaced vertices between the almost degenerate pseudo-Dirac neutralinos are a smoking gun signature of such a framework. However, in our case, the primary decay modes are $h \nu_{i}, Z \nu$ and $W^{ \pm} \ell^{\mp}$ and the decay to gravitino is largely suppressed. In fact, a detailed study of the trilinear terms makes our scenario phenomenologically distinct, rich and explorable at the LHC.

\section{Conclusion}

Neutrinos and dark matter play a very important role in understanding the physics beyond the SM. Supersymmetric models while solving the hierarchy problem can also address the issues pertaining to neutrino masses and dark matter. However, the present searches by both ATLAS and CMS have found no significant excess in their pursuit of superpartners. As a result, stringent constraints are obtained on the superpartner masses. In this light, models with $R$-symmetry and Dirac gauginos are well motivated as they can relax these constraints. Therefore, in this paper, we come up with an $R$-symmetric SUSY scenario with specific $R$-charges leading to bilinear and trilinear " $R$-parity violating" terms at the superpotential. Our prime aim in this paper is to explain how active light neutrino masses and mixing can be generated. In the process we also discuss the generation of a Higgs mass around $125 \mathrm{GeV}$. Then we motivate the requirement of $R$-symmetry breaking through anomaly mediation. The bilinear terms from the superpotential and the scalar potential can be rotated away simultaneously due to the suitable choice of basis. However, the trilinear terms will always be there playing an important role in generating neutrino masses and mixing. We provide 
constraints on the relevant superpotential parameters while fitting neutrino masses for both normal and inverted hierarchies. In our scenario neutrino masses are generated at the tree level itself which is vastly different than the standard RPV-MSSM scenarios. In standard RPV-MSSM scenario, lepton number violation can emerge from the bilinear $\left(\epsilon_{i} \widehat{H}_{u} \widehat{L}_{i}\right)$ as well as the trilinear $\left(\lambda_{i j k} \widehat{L}_{i} \widehat{L}_{j} \widehat{E}_{K}^{c}, \lambda_{i j k}^{\prime} \widehat{L}_{i} \widehat{Q}_{j} \widehat{D}_{K}^{c}\right)$ terms in the superpotential. In case of bilinear RPV, only one of the neutrinos acquire mass at the tree level, while the other two become massive through one-loop induced effects. But such a process necessitates significant tuning between the model parameters. Again, in presence of trilinear RPV, all the neutrinos acquire masses at the one-loop level. As these operators are constrained from lepton flavor violating (LFV) processes, a minuscule room is left to fit neutrino masses and mixing after satisfying all other constraints. Unlike usual scenarios, in our framework, neutrino masses and mixing can be explained at the tree level itself even with not so finetuned values of the superpotential parameters $\xi, \eta \sim \mathcal{O}\left(10^{-3}-10^{-4}\right)$. In passing we would like to mention that such choice of parameters are also compatible with LFV constraints.

In our proposal, LSP gravitino mass turns out to be the order parameter of $R$-breaking and it qualifies to be an excellent DM candidate. We explore this possibility by considering the production and decays of gravitino. While incorporating the constraints from diffuse gamma ray experiment, the model becomes more predictive leaving an allowed region of the gravitino mass in the range $200 \mathrm{keV} \lesssim m_{3 / 2} \lesssim 0.1 \mathrm{GeV}$. The collider signatures are also quite different from the standard RPV-MSSM case. In our framework, lightest neutralino decay leads to di-Higgs signatures. Similarly, pair-production of charginos may also lead to a pair of Higgs accompanied by opposite signed leptons. These decays are controlled by the superpotential parameters $\xi, \eta$ and for their suitable values $\sim \mathcal{O}\left(10^{-3}\right)$ prompt decay of electro-weakinos could be observed.

\section{Acknowledgments}

We thank Sourov Roy for many helpful discussions and insights. SC would also like to thank the hospitality of IIT Kanpur and HRI Allahabad where a part of the project was completed. Work of J.C. is supported by the Department of Science and Technology, Government of India, under the Grant IFA12-PH-34 (INSPIRE Faculty Award); and the Science and Engineering Research Board, Government of India, under the agreement SERB/PHY/2016348 (Early Career Research Award).

Open Access. This article is distributed under the terms of the Creative Commons Attribution License (CC-BY 4.0), which permits any use, distribution and reproduction in any medium, provided the original author(s) and source are credited.

\section{References}

[1] I. Esteban, M.C. Gonzalez-Garcia, M. Maltoni, I. Martinez-Soler and T. Schwetz, Updated fit to three neutrino mixing: exploring the accelerator-reactor complementarity, JHEP 01 (2017) 087 [arXiv: 1611.01514] [INSPIRE].

[2] Planck collaboration, P.A.R. Ade et al., Planck 2013 results. XVI. Cosmological parameters, Astron. Astrophys. 571 (2014) A16 [arXiv:1303.5076] [INSPIRE]. 
[3] A.C.M. Correia et al., The HARPS search for southern extra-solar planets XIX. Characterization and dynamics of the GJ876 planetary system, Astron. Astrophys. 511 (2010) A21 [arXiv:1001.4774] [INSPIRE].

[4] R. Barbier et al., R-parity violating supersymmetry, Phys. Rept. 420 (2005) 1 [hep-ph/0406039] [INSPIRE].

[5] J.L. Feng, Dark Matter Candidates from Particle Physics and Methods of Detection, Ann. Rev. Astron. Astrophys. 48 (2010) 495 [arXiv: 1003.0904] [INSPIRE].

[6] ATLAS collaboration, Further searches for squarks and gluinos in final states with jets and missing transverse momentum at $\sqrt{s}=13 \mathrm{TeV}$ with the ATLAS detector, ATLAS-CONF-2016-078 (2016).

[7] CMS collaboration, Search for supersymmetry in events with jets and missing transverse momentum in proton-proton collisions at 13 TeV, CMS-PAS-SUS-16-014 (2016).

[8] P.J. Fox, A.E. Nelson and N. Weiner, Dirac gaugino masses and supersoft supersymmetry breaking, JHEP 08 (2002) 035 [hep-ph/0206096] [INSPIRE].

[9] G. Grilli di Cortona, E. Hardy and A.J. Powell, Dirac vs Majorana gauginos at a $100 \mathrm{TeV}$ collider, JHEP 08 (2016) 014 [arXiv: 1606.07090] [INSPIRE].

[10] G.D. Kribs and A. Martin, Supersoft Supersymmetry is Super-Safe, Phys. Rev. D 85 (2012) 115014 [arXiv: 1203.4821] [INSPIRE].

[11] G.D. Kribs, E. Poppitz and N. Weiner, Flavor in supersymmetry with an extended R-symmetry, Phys. Rev. D 78 (2008) 055010 [arXiv:0712.2039] [INSPIRE].

[12] C. Frugiuele and T. Gregoire, Making the Sneutrino a Higgs with a $\mathrm{U}(1)_{R}$ Lepton Number, Phys. Rev. D 85 (2012) 015016 [arXiv:1107.4634] [INSPIRE].

[13] E. Bertuzzo and C. Frugiuele, Fitting Neutrino Physics with a $\mathrm{U}(1)_{R}$ Lepton Number, JHEP 05 (2012) 100 [arXiv: 1203.5340] [INSPIRE].

[14] C. Frugiuele, T. Gregoire, P. Kumar and E. Ponton, ' $L=R$ ' $-\mathrm{U}(1)_{R}$ as the Origin of Leptonic 'RPV', JHEP 03 (2013) 156 [arXiv:1210.0541] [INSPIRE].

[15] C. Frugiuele, T. Gregoire, P. Kumar and E. Ponton, ' $L=R$ ' - U(1) $R$ Lepton Number at the LHC, JHEP 05 (2013) 012 [arXiv:1210.5257] [INSPIRE].

[16] S. Chakraborty and S. Roy, Higgs boson mass, neutrino masses and mixing and keV dark matter in an $\mathrm{U}(1)_{R}-$ lepton number model, JHEP 01 (2014) 101 [arXiv:1309.6538] [INSPIRE].

[17] S. Chakraborty, D.K. Ghosh and S. Roy, $7 \mathrm{keV}$ Sterile neutrino dark matter in $\mathrm{U}(1)_{R}$-lepton number model, JHEP 10 (2014) 146 [arXiv:1405.6967] [INSPIRE].

[18] S. Chakraborty, A. Datta and S. Roy, $h \rightarrow \gamma \gamma$ in $\mathrm{U}(1)_{R}$-lepton number model with a right-handed neutrino, JHEP 02 (2015) 124 [Erratum ibid. 09 (2015) 077] [arXiv: 1411.1525] [INSPIRE].

[19] S. Chakraborty, A. Datta, K. Huitu, S. Roy and H. Waltari, Light top squarks in $\mathrm{U}(1)_{R}$-lepton number model with a right handed neutrino and the LHC, Phys. Rev. D 93 (2016) 075005 [arXiv: 1508.01875] [INSPIRE].

[20] P. Coloma and S. Ipek, Neutrino masses from a pseudo-Dirac Bino, Phys. Rev. Lett. 117 (2016) 111803 [arXiv:1606.06372] [INSPIRE]. 
[21] R. Fok and G.D. Kribs, $\mu$ to e in R-symmetric Supersymmetry, Phys. Rev. D 82 (2010) 035010 [arXiv: 1004.0556] [INSPIRE].

[22] S.Y. Choi, D. Choudhury, A. Freitas, J. Kalinowski, J.M. Kim and P.M. Zerwas, Dirac Neutralinos and Electroweak Scalar Bosons of $N=1 / N=2$ Hybrid Supersymmetry at Colliders, JHEP 08 (2010) 025 [arXiv:1005.0818] [INSPIRE].

[23] R. Fok, G.D. Kribs, A. Martin and Y. Tsai, Electroweak Baryogenesis in R-symmetric Supersymmetry, Phys. Rev. D 87 (2013) 055018 [arXiv:1208.2784] [INSPIRE].

[24] S. Chakraborty, A. Chakraborty and S. Raychaudhuri, Diphoton resonance at $750 \mathrm{GeV}$ in the broken R-symmetric MSSM, Phys. Rev. D 94 (2016) 035014 [arXiv:1512.07527] [INSPIRE].

[25] K. Benakli, M.D. Goodsell and F. Staub, Dirac Gauginos and the 125 GeV Higgs, JHEP 06 (2013) 073 [arXiv: 1211.0552] [INSPIRE].

[26] M.D. Goodsell, M.E. Krauss, T. Müller, W. Porod and F. Staub, Dark matter scenarios in a constrained model with Dirac gauginos, JHEP 10 (2015) 132 [arXiv:1507.01010] [INSPIRE].

[27] A.E. Nelson and T.S. Roy, New Supersoft Supersymmetry Breaking Operators and a Solution to the $\mu$ Problem, Phys. Rev. Lett. 114 (2015) 201802 [arXiv:1501.03251] [InSPIRE].

[28] M.D. Goodsell, Two-loop RGEs with Dirac gaugino masses, JHEP 01 (2013) 066 [arXiv: 1206.6697] [INSPIRE].

[29] Z. Chacko, M.A. Luty, I. Maksymyk and E. Ponton, Realistic anomaly mediated supersymmetry breaking, JHEP 04 (2000) 001 [hep-ph/9905390] [INSPIRE].

[30] G.G. Ross and J.W.F. Valle, Supersymmetric Models Without R-Parity, Phys. Lett. B 151 (1985) 375 [INSPIRE].

[31] J.R. Ellis, G. Gelmini, C. Jarlskog, G.G. Ross and J.W.F. Valle, Phenomenology of Supersymmetry with Broken R-Parity, Phys. Lett. B 150 (1985) 142 [INSPIRE].

[32] F. Bazzocchi, S. Morisi, E. Peinado, J.W.F. Valle and A. Vicente, Bilinear R-parity violation with flavor symmetry, JHEP 01 (2013) 033 [arXiv:1202.1529] [INSPIRE].

[33] Y. Grossman and S. Rakshit, Neutrino masses in R-parity violating supersymmetric models, Phys. Rev. D 69 (2004) 093002 [hep-ph/0311310] [InSPIRE].

[34] Y. Grossman and H.E. Haber, Sneutrino mixing phenomena, Phys. Rev. Lett. 78 (1997) 3438 [hep-ph/9702421] [INSPIRE].

[35] T. Banks, Y. Grossman, E. Nardi and Y. Nir, Supersymmetry without R-parity and without lepton number, Phys. Rev. D 52 (1995) 5319 [hep-ph/9505248] [INSPIRE].

[36] M. Bisset, O.C.W. Kong, C. Macesanu and L.H. Orr, A simple phenomenological parametrization of supersymmetry without R-parity, Phys. Lett. B 430 (1998) 274 [hep-ph/9804282] [INSPIRE].

[37] P. Binetruy, E. Dudas, S. Lavignac and C.A. Savoy, Quark flavor conserving violations of the lepton number, Phys. Lett. B 422 (1998) 171 [hep-ph/9711517] [INSPIRE].

[38] Y. Grossman and H.E. Haber, (S)neutrino properties in R-parity violating supersymmetry. 1. CP conserving phenomena, Phys. Rev. D 59 (1999) 093008 [hep-ph/9810536] [INSPIRE].

[39] P. Dießner, J. Kalinowski, W. Kotlarski and D. Stöckinger, Higgs boson mass and electroweak observables in the MRSSM, JHEP 12 (2014) 124 [arXiv:1410.4791] [INSPIRE]. 
[40] P. Diessner, J. Kalinowski, W. Kotlarski and D. Stöckinger, Two-loop correction to the Higgs boson mass in the MRSSM, Adv. High Energy Phys. 2015 (2015) 760729.

[41] M. Hirsch, M.A. Diaz, W. Porod, J.C. Romao and J.W.F. Valle, Neutrino masses and mixings from supersymmetry with bilinear $R$ parity violation: A theory for solar and atmospheric neutrino oscillations, Phys. Rev. D 62 (2000) 113008 [Erratum ibid. D 65 (2002) 119901] [hep-ph/0004115] [INSPIRE].

[42] M.A. Diaz, M. Hirsch, W. Porod, J.C. Romao and J.W.F. Valle, Solar neutrino masses and mixing from bilinear $R$ parity broken supersymmetry: Analytical versus numerical results, Phys. Rev. D 68 (2003) 013009 [Erratum ibid. D 71 (2005) 059904] [hep-ph/0302021] [INSPIRE].

[43] Mu2E collaboration, L. Bartoszek et al., Mu2e Technical Design Report, arXiv:1501.05241 [INSPIRE].

[44] M.B. Gavela, T. Hambye, D. Hernández and P. Hernández, Minimal Flavour Seesaw Models, JHEP 09 (2009) 038 [arXiv:0906.1461] [INSPIRE].

[45] M. Bolz, A. Brandenburg and W. Buchmüller, Thermal production of gravitinos, Nucl. Phys. B 606 (2001) 518 [Erratum ibid. B 790 (2008) 336] [hep-ph/0012052] [INSPIRE].

[46] J. Pradler and F.D. Steffen, Thermal gravitino production and collider tests of leptogenesis, Phys. Rev. D 75 (2007) 023509 [hep-ph/0608344] [INSPIRE].

[47] N.E. Bomark and L. Roszkowski, $3.5 \mathrm{keV}$ x-ray line from decaying gravitino dark matter, Phys. Rev. D 90 (2014) 011701 [arXiv:1403.6503] [INSPIRE].

[48] M. Fujii, M. Ibe and T. Yanagida, Upper bound on gluino mass from thermal leptogenesis, Phys. Lett. B 579 (2004) 6 [hep-ph/0310142] [INSPIRE].

[49] E.A. Baltz and H. Murayama, Gravitino warm dark matter with entropy production, JHEP 05 (2003) 067 [astro-ph/0108172] [INSPIRE].

[50] M. Fujii and T. Yanagida, Natural gravitino dark matter and thermal leptogenesis in gauge mediated supersymmetry breaking models, Phys. Lett. B 549 (2002) 273 [hep-ph/0208191] [INSPIRE].

[51] F. Takayama and M. Yamaguchi, Gravitino dark matter without R-parity, Phys. Lett. B 485 (2000) 388 [hep-ph/0005214] [INSPIRE].

[52] R. Essig, E. Kuflik, S.D. McDermott, T. Volansky and K.M. Zurek, Constraining Light Dark Matter with Diffuse X-Ray and Gamma-Ray Observations, JHEP 11 (2013) 193 [arXiv:1309.4091] [INSPIRE].

[53] L. Bouchet et al., INTEGRAL SPI All-Sky View in Soft Gamma Rays: Study of Point Source and Galactic Diffuse Emissions, Astrophys. J. 679 (2008) 1315 [arXiv:0801.2086] [INSPIRE].

[54] G. Weidenspointner et al., The comptel instrumental line background, AIP Conf. Proc. 510 (2004) 581 [astro-ph/0012332] [INSPIRE].

[55] EGRET collaboration, P. Sreekumar et al., EGRET observations of the extragalactic gamma-ray emission, Astrophys. J. 494 (1998) 523 [astro-ph/9709257] [INSPIRE].

[56] Fermi-LAT collaboration, M. Ackermann et al., Fermi-LAT Observations of the Diffuse Gamma-Ray Emission: Implications for Cosmic Rays and the Interstellar Medium, Astrophys. J. 750 (2012) 3 [arXiv:1202.4039] [INSPIRE]. 
[57] J.F. Navarro, C.S. Frenk and S.D.M. White, The structure of cold dark matter halos, Astrophys. J. 462 (1996) 563 [astro-ph/9508025] [INSPIRE].

[58] J.F. Navarro, C.S. Frenk and S.D.M. White, A universal density profile from hierarchical clustering, Astrophys. J. 490 (1997) 493 [astro-ph/9611107] [INSPIRE].

[59] W. Fischler, G.F. Giudice, R.G. Leigh and S. Paban, Constraints on the baryogenesis scale from neutrino masses, Phys. Lett. B 258 (1991) 45 [INSPIRE].

[60] B.A. Campbell, S. Davidson, J.R. Ellis and K.A. Olive, Cosmological baryon asymmetry constraints on extensions of the standard model, Phys. Lett. B 256 (1991) 484 [INSPIRE].

[61] H.K. Dreiner and G.G. Ross, Sphaleron erasure of primordial baryogenesis, Nucl. Phys. B 410 (1993) 188 [hep-ph/9207221] [INSPIRE].

[62] V.A. Kuzmin, V.A. Rubakov and M.E. Shaposhnikov, On the Anomalous Electroweak Baryon Number Nonconservation in the Early Universe, Phys. Lett. B 155 (1985) 36 [INSPIRE].

[63] P. Kumar and E. Ponton, Electroweak Baryogenesis and Dark Matter with an approximate R-symmetry, JHEP 11 (2011) 037 [arXiv:1107.1719] [INSPIRE].

[64] R. Fok, G.D. Kribs, A. Martin and Y. Tsai, Electroweak Baryogenesis in R-symmetric Supersymmetry, Phys. Rev. D 87 (2013) 055018 [arXiv: 1208.2784] [INSPIRE].

[65] I. Affleck and M. Dine, A New Mechanism for Baryogenesis, Nucl. Phys. B 249 (1985) 361 [INSPIRE].

[66] S. Bobrovskyi, J. Hajer and S. Rydbeck, Long-lived higgsinos as probes of gravitino dark matter at the LHC, JHEP 02 (2013) 133 [arXiv:1211.5584] [INSPIRE].

[67] S. Biswas, E.J. Chun and P. Sharma, Di-Higgs signatures from R-parity violating supersymmetry as the origin of neutrino mass, JHEP 12 (2016) 062 [arXiv:1604.02821] [INSPIRE].

[68] B. Mukhopadhyaya, S. Roy and F. Vissani, Correlation between neutrino oscillations and collider signals of supersymmetry in an R-parity violating model, Phys. Lett. B 443 (1998) 191 [hep-ph/9808265] [INSPIRE].

[69] E.J. Chun, CP violation, sneutrino oscillation and neutrino masses in R-parity violating supersymmetric standard model, Phys. Lett. B 525 (2002) 114 [hep-ph/0105157] [INSPIRE].

[70] S. Roy and B. Mukhopadhyaya, Some implications of a supersymmetric model with R-parity breaking bilinear interactions, Phys. Rev. D 55 (1997) 7020 [hep-ph/9612447] [INSPIRE].

[71] Y. Grossman, B. Shakya and Y. Tsai, Neutralino Oscillations at the LHC, Phys. Rev. D 88 (2013) 035026 [arXiv:1211.3121] [INSPIRE]. 